\title{
DE CÓMO LA REALIDAD PUEDE TOMAR PARTE EN JUEGOS EMERGENTES
}

Federico Peinado y Michael Santorum

\section{Resumen}

Este artículo es un estudio del fenómeno de la utilización de elementos reales en aplicaciones software de entretenimiento. De manera más o menos explícita y significativa, este fenómeno se encuentra en la experiencia de juego de numerosos juguetes y experimentos digitales, así como de algunos videojuegos comerciales de gran popularidad; es interesante conocer, por tanto, sus implicaciones estéticas y funcionales a la hora de diseñar interfaces y sistemas de jugabilidad emergente, así como la posible evolución que este fenómeno de la realidad como componente lúdico puede sufrir en los próximos años.

\section{Palabras clave}

videojuegos - diseño - realidad-emergente

\section{ON HOW REALITY CAN TAKE PART IN EMERGENT GAMES}

Michael Santorum and Federico Peinado

\begin{abstract}
This paper is a study of the phenomenon of using real elements in entertainment software applications. In a more or less explicit and significant way, this phenomenon is found in the game experience of many digital toys and experiments, as in some commercial videogames of great popularity; it is interesting to know, therefore, its nature, its aesthetic and functional implications when designing interfaces and systems of emergent playability, and also the possible evolution that this phenomenon of reality as recreational component could go through during next years.
\end{abstract}

\section{Keywords}

$$
\text { videogames - design - emergent-reality }
$$




\section{Introducción}

Los juegos representan una oportunidad de aprender a interactuar con la realidad de una manera simplificada, pero divertida y segura. El jugador puede enfrentarse con situaciones ficticias que le dan la oportunidad de probar diferentes soluciones a un problema sin la tensión y el riesgo que conllevaría la misma situación en el mundo real. La posibilidad de equivocarse sin temor a un castigo y la oportunidad de repetir la misma situación infinidad de veces, permite al usuario pensar soluciones nuevas y adaptarlas en función de los resultados obtenidos, haciendo de la ficción un excelente campo de entrenamiento.

Jugando con las palabras podría argumentarse que no existe nada "realmente” ficticio en este mundo. Siendo real toda existencia efectiva y siendo dicha existencia lo único que verdaderamente conocemos, todos los artificios del ser humano están articulados en función de lo real, aunque sólo sea como referencia esencial que podemos seguir fielmente, distorsionar, contradecir e incluso intentar ignorar por completo.

Si aceptamos lo anterior, la ficción de la que forman parte los juegos tampoco puede escapar de hacer referencia constante a la realidad. Además de los elementos “propiamente reales” que toda definición de juego requiere (los jugadores, la plataforma, los creadores, etc.), el poder creciente de la Informática permite que los videojuegos utilicen entornos virtuales cada vez más realistas, donde se simula el gobierno de las leyes de la Lógica y la Física, donde la naturaleza, el clima e incluso la sociedad y la inteligencia de sus individuos parecen vivos retratos de la auténtica naturaleza, clima, sociedad e inteligencia de sus individuos, y donde también es posible encontrar réplicas explícitas y precisas a acontecimientos y personajes que forman parte de nuestra Historia.

Sin embargo todas estas referencias de las que hablamos son escogidas y procesadas por los creadores del juego durante la etapa plenamente consciente, meticulosa y laboriosa de diseño. En esta etapa es donde resulta innegable la influencia de lo real, aunque por otro lado, una vez concluida la creación del juego, los elementos reales que han sido utilizados durante el proceso quedan “encapsulados” dentro de la ficción y pasan a tener un rol pasivo. En este punto de nuestro discurso es cuando sacamos a relucir que existen algunos casos, los más interesantes para este estudio, donde los elementos reales (más allá de los jugadores, la plataforma, los creadores, etc.) toman parte activa y significativa en el desarrollo de un videojuego, sin haber sido procesados o prefabricados previamente por los diseñadores, y generando de manera emergente nuevas posibilidades de juego.

\section{La naturaleza del fenómeno}

Crawford (1982) habla de la "representación de la realidad" como uno de las cuatro características fundamentales de todo juego, junto con la interacción, el conflicto y la seguridad. Sin embargo en la introducción de este estudio hemos aclarado que sólo se considerarán aquellos elementos que no sólo se 
“representan” en el juego sino que participan de forma activa en su desarrollo. Para poder estudiar este fenómeno es necesario considerar un amplio abanico de posibles elementos reales que pueden servir para generar jugabilidad emergente.

\section{Historia y actualidad}

En muchos juegos de guerra, las misiones son conocidas batallas, lo que ahorra a los desarrolladores proporcionar al jugador información adicional sobre el contexto del conflicto. La imaginación del jugador rellenará con todo lujo de detalles lo que quede fuera de su campo de visión o no aparezca en el juego, utilizando los recuerdos de situaciones similares que tenga en su memoria.

Por ejemplo, en el juego Call of Duty 2, el jugador interpreta cuatro personajes donde cada uno de ellos protagoniza misiones históricas ejecutadas durante la Segunda Guerra Mundial:

- Soldado Vasili Ivanovich Koslov, $13^{\circ}$ División de Fusileros del Ejercito Rojo: Batalla de Moscú, diciembre de 1941; Batalla de Estalingrado, diciembre de 1942 y febrero de 1943.

- Sargento John Davis, $7^{a}$ División Acorazada del Ejercito Británico: 2a Batalla de El Alamein, octubre de 1942; Campaña de Túnez, marzo de 1943; Batalla de Normandía, junio de 1944.

- Comandante David Welsh, 7ª División Acorazada del Ejercito Británico: Libia, enero 1943.

- Cabo Bill Taylor, $2^{\circ}$ Batallón de la Armada de los Estados Unidos: Point du Hoc, Normandía, junio de 1944; Batalla del Bosque de Hurtgen, Colina 400, diciembre 1944; Rin, marzo 1945.

Aunque estas referencias históricas se toman durante la etapa de diseño, a veces la actualización de los contenidos y el estreno de nuevas versiones es tan veloz que se consigue la sensación de que la realidad forme parte del juego. Este es el caso de Kuma|War, donde los diseñadores se nutren de noticias sobre conflictos armados actuales para crear juegos de guerra. Su lema es “real war news, real war games”.

Si bien es cierto que existen juegos más “convencionales” que también actualizan su trasfondo para adaptarlo a escenarios bélicos contemporáneos (Ej. invasión de Irak en Battlefield 1942, Desert Combat) lo cierto es que pocos se adaptan, como KumalWar, a un ritmo casi semanal a las decenas de eventos significativos que ocurren en estos conflictos.

El último ejemplo que podemos encontrar es Paris Riots, una adaptación del juego de EA Medal of Honor para recrear las revueltas vividas en Francia a finales del año 2005, en las cuales se enfrentaron miles de ciudadanos franceses a las fuerzas de seguridad de estado.

Por otra parte, gracias al empuje de ciertos grupos de investigación en "serious games” y aplicaciones publicitarias, propagandísticas, etc. de los videojuegos, temas como la política o la religión cobran cada vez mayor importancia en el diseño de videojuegos. Pueden encontrarse ejemplos de activismo político o 
ideológico en títulos como September 12th, Food Force o McDonalds Videogame, así como títulos de temática religiosa como Catechumen.

Dejando a un lado la guerra y las ideologías, el género deportivo también se adapta (como mínimo anualmente) para reflejar en la ficción del juego los acontecimientos deportivos reales. Las bases de datos que utilizan los títulos de la serie EA Sports están repletas de datos reales, actualizados antes de cada liga o campeonato, siendo generalmente los usuarios los que van más allá y en ocasiones, gracias al juego en red, se encargan de introducir en el juego, día a día, cada nuevo detalle que da a conocer la prensa deportiva.

\section{Cultura}

El cine o la literatura generan ficciones que a menudo se convierten en fenómenos mediáticos y sociales que influyen en los videojuegos, o determinan completamente su contenido (como en el caso de las licencias). Las películas, por ejemplo, han creado clichés fácilmente reconocibles que ayudan a revelar con unos pocos trazos la personalidad de un individuo, la información necesaria para identificar un lugar o una situación, etc. Gracias a estos clichés, el jugador se encuentra con representaciones de eventos que, sin ser reales, resultan creíbles y se vuelven perfectamente plausibles en su imaginación. Por ejemplo, la experiencia de combate del soldado Vasili Ivanovich Koslov en la ciudad de Estalingrado resultará mucho más creíble para el jugador si ha visto la película Enemigo a las puertas (Annaud, 2001).

La utilización de la cultura mediática del jugador para incrementar la calidad de la experiencia de juego ha sido una constante desde el nacimiento de los videojuegos. La escasa calidad visual de los primeros títulos obligaba a los desarrolladores a exprimir al máximo la imaginación del jugador para situarlos en un entorno creíble; para ello era necesario un proceso de “enajenación” voluntaria por parte del jugador que, gracias a los avances en informática gráfica de los nuevos títulos, casi ha desaparecido. Las dos imágenes que mostramos a continuación dejan clara esta evolución:

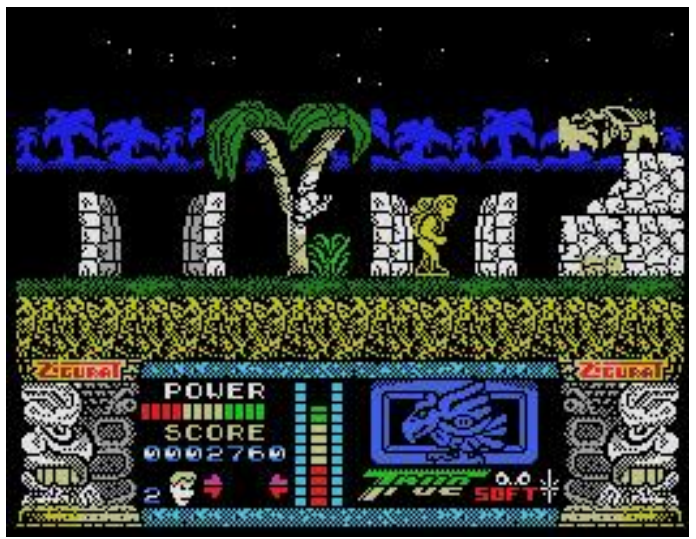

Acción en la jungla, según Jungle Warrior, 1990

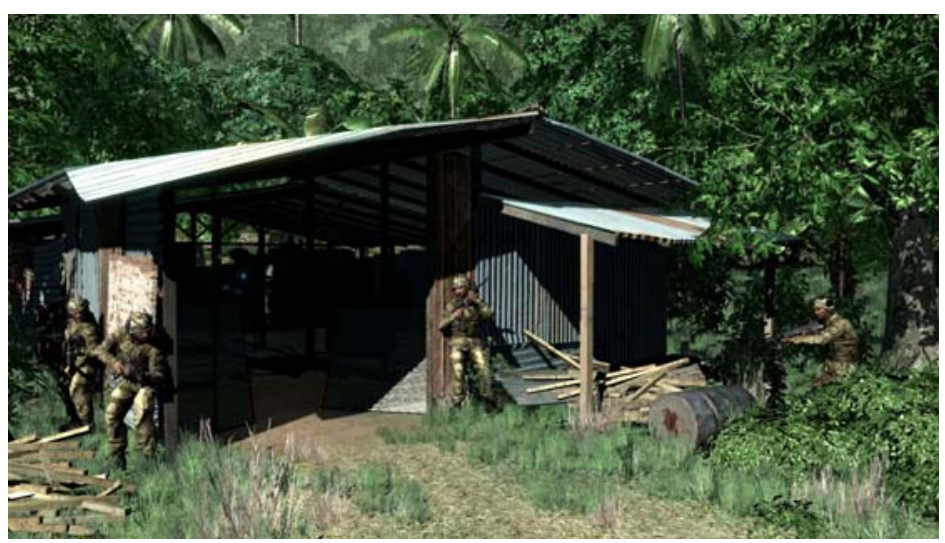

Acción en la jungla, según Crysis, previsto para 2007 
Una vez alcanzado el “realismo” visual, la jugabilidad cobra mayor importancia, siendo la simulación del comportamiento de la realidad (más que su aspecto) y el diseño de interfaces más naturales e inmersivas son los siguientes objetivos. Es en este punto donde, de nuevo, las formas narrativas más clásicas ayudan a crear mecanismos de juego más sencillos que el jugador puede aprender a usar rápidamente. Simular la realidad en todas sus facetas es una utopía, y además no es razonable, ya que desde el punto de vista de un juego es más importante lograr un entorno simplificado, aunque creíble y que permita una buena jugabilidad, que un entorno realista. La experiencia fantástica de ver un capítulo de una serie policíaca se aproxima más al objetivo deseado para un videojuego que la experiencia cotidiana de una pareja de policías reales.

Si bien Tetris es un juego abstracto de puzzles, con un comportamiento muy sencillo de aprender, Grand Theft Auto: San Andreas tiene multitud de comportamientos similares a los de la vida real que no necesitan de aprendizaje por parte del jugador, ya que los conoce de la vida cotidiana y sobre todo de las películas que está acostumbrado a ver. Juegos como Half-Life 2 cuentan además con un trasfondo narrativo externo al juego, repartido en guías y sitios web, que permite a los jugadores más curiosos completar la información del juego y la serie Myst generó una saga de libros complementarios para explicar el mundo en el que se desarrollaba.

\section{Calendario y condiciones meteorológicas}

Desde el punto de vista del jugador todos los juegos discurren en tiempo real, sin embargo es dentro de la ficción del juego donde el tiempo puede estructurarse de muy diversas formas, siendo su relación con el tiempo real la que crea todas las implicaciones narrativas que tienen también las obras literarias o cinematográficas. En Shadow of Memories el protagonista dispone de tan solo unas horas para resolver su propio crimen. En la trama los viajes en el tiempo, las paradojas y las elipsis se realizan para acelerar el curso de la historia y hacer más interesante y atractivo el argumento, siendo el tiempo un recurso limitado que el jugador debe gestionar adecuadamente si quiere resolver los enigmas del juego.

Algo más inusual es lo que ocurre en Animal Crossing, donde el tiempo discurre de forma exacta fuera del juego como dentro de él; y no sólo las horas sino los días, los meses y hasta incluso las estaciones del año. La noche es oscura, las hojas de los árboles caen al llegar el otoño... en definitiva: el juego responde fielmente al calendario real que forma parte de la vida del jugador.

Otros títulos no contienen elementos sustanciales pero sí sutiles detalles que integran la vida real en el juego. Este es el caso de World of Warcraft donde el entorno cambia en épocas señaladas, engalanándose correspondientemente en fechas señaladas como Halloween o Nochebuena; de hecho a menudo los creadores del juego diseñan nuevas misiones específicamente para conmemorar esas fechas, contando el juego con un calendario de eventos de hasta con un año de antelación. 
El simulador de vuelo Flight Simulator 2004. A Century of Flight utiliza datos de elevación reales para crear el entorno y una física de vuelo simplificada para dar al jugador la sensación de volar. El juego permite crear condiciones climatológicas a la carta o conectarse a un servidor de Internet para jugar en tiempo real con los datos metereológicos concretos que se dan en una región concreta del mundo, permitiendo que en el mismo espacio se deba reaccionar de diferente forma dependiendo del tiempo existente, manteniendo la misma premisa de juego. La realidad puede ayudar a crear nuevos retos manteniendo siempre una estructura narrativa básica: ir de un punto a otro puede ser un agradable paseo en avioneta o una lucha contra los elementos que exige del jugador toda su destreza para alcanzar el destino.

\section{Movimiento y emociones}

Aunque el jugador es uno de esos elementos de la realidad de los que hemos prescindido en este estudio, su estado emocional o físico no se considera habitualmente parte de la interfaz con el juego. En ciertos juegos experimentales que utilizan tecnologías de Computación Afectiva (Guilleade et al. 2005) es posible recibir realimentación biológica del jugador para identificar las emociones que siente un jugador en cada momento del juego. Por ejemplo la conductividad eléctrica de su piel puede darnos una idea del estrés a que está sometido el jugador y esta información puede utilizarse para que el juego se vuelva más fácil o difícil en función del autocontrol y la calma del jugador.

Muchos de estos prototipos experimentales han sido comercializados ya. Hoy día las consolas de última generación incorporan dispositivos para capturar el movimiento o la imagen de un jugador (como por ejemplo una cámara, una alfombra de baile u otros periféricos similares). Podemos entender que estos y otros dispositivos más avanzados formarán parte del interfaz estándar de los videojuegos del futuro, sin embargo no dejan de ser posibilidades para ir incorporando elementos de la realidad en el juego.

\section{Realidad aumentada}

Canyouseemenow, es un proyecto en el cual se mezcla realidad y juego por Internet. Existen cinco corredores equipados con PDAs con sistema GPS para determinar su posición geográfica, que se refleja en todo momento en un mapa visualizable desde el sitio web del juego. Los usuarios pueden conectarse a este sitio web y jugar a atrapar a los corredores desde su casa, moviendo su avatar con las teclas de dirección, o bien a subir de los corredores reales, que a su vez perseguirán las representaciones de los jugadores que aparecen en sus PDAs. Cuando uno de los corredores se acerca a menos de cinco metros de la representación de uno de los jugadores, este se considera atrapado, se saca una foto del lugar real en el cual se ha producido la “captura” y se acaba el juego para ese jugador. 
La separación existente entre los perseguidores y los perseguidos utiliza las fotografías de los atrapados para dotar de mayor realidad al mapa virtual del juego, que no puede representar fielmente las calles por las que se realiza el juego, llenos de gente ajena al juego, coches, semáforos...
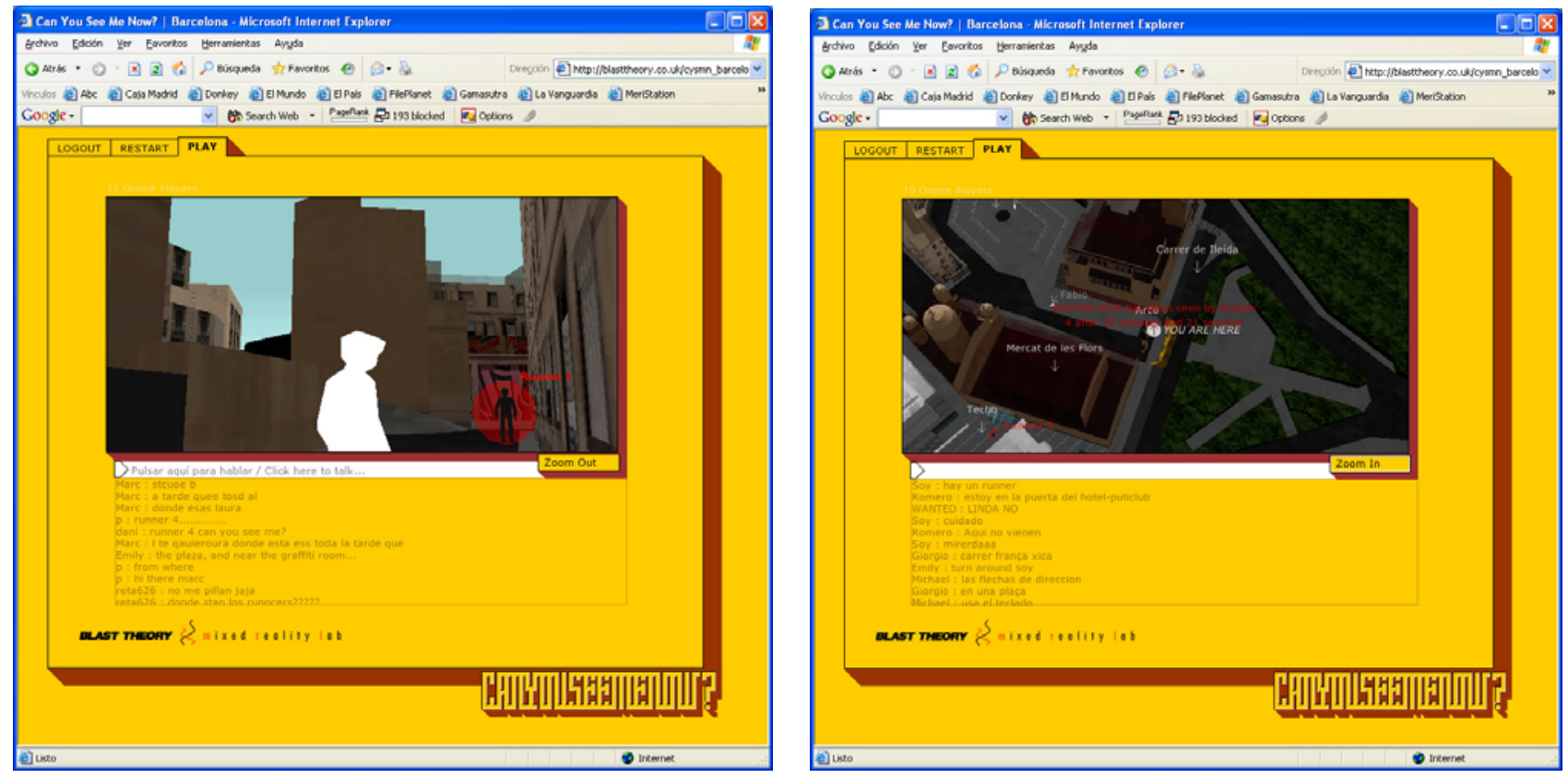

\section{Relaciones sociales, laborales y económicas}

Estos factores resultan sumamente importantes en los videojuegos modernos, aunque son ciertamente más difíciles de identificar como elementos reales que toman parte en la jugabilidad emergente. Todo juego establece una cierta relación real entre sus jugadores. Los juegos en ocasiones se utilizan para "romper el hielo” o como una manera de comparar nuestras habilidades con las de otra persona. Pero en algunos casos concretos, como en el del simulador de imperios intergalácticos oGame, la adicción y el sistema de “castas” que genera el juego en los jugadores es la base lógica y comercial (ya que los creadores sólo cobran por la publicidad de su sitio web) que sustenta todo el juego.

En juegos como EverQuest o el clásico Ultima Online se llegaron a crear verdaderos sistemas económicos, donde se compran y venden bienes virtuales ligados al juego. Lo que ocurre, aunque en estos casos el tema suele estar muy perseguido por las empresas afectadas, es que se establecen leyes de intercambio entre moneda real y virtual, generando por lo tanto un sistema económico completamente real.

En China y Core existen la figura del jugador profesional que cobra un sueldo a cambio de mejorar y otorgar experiencia a los personajes controlados por otros jugadores clientes, como ha ocurrido en World of Warcraft. Especialmente en el género de los juegos de multijugador masivo en red (cuyas siglas en inglés son MMOGs) es donde las relaciones sociales, laborales y económicas de los jugadores generan jugabilidad emergente. Y el futuro nos depara relaciones afectivas virtuales, como las de V-Girl, un juego para móviles 
con parejas virtuales a las que tendremos mantener contentas comprando regalos reales, llamando por teléfono a todas horas, etc.

\section{Implicaciones funcionales y estéticas}

A la hora de diseñar un videojuego es importante tener en cuenta que ventajas e inconvenientes puede tener el incorporar elementos reales en sus mecanismos de funcionamiento. Existen implicaciones funcionales pero también estéticas relacionadas con esta decisión, siendo ambas igual de importantes para el diseño de un juego. En este apartado tratamos de analizar algunas de ellas:

\section{Realidad e interfaz}

Habitualmente todo lo referente a la interfaz que utiliza el jugador para comunicarse con los elementos ficticios del juego se considera extensión de este y por lo tanto no interesa de cara a este análisis. Sin embargo como se dijo en el apartado de movimiento y emociones, hay buenos ejemplos de excepciones a esta norma, donde se rompen las convenciones establecidas con el jugador y se otorga una nueva dimensión a la interfaz, haciendo notar su condición de “elemento real” aparte de una extensión natural del jugador.

En Metal Gear Solid, algo tan sencillo como la vibración del controlador del juego se utiliza para dar un "masaje” al protagonista (en realidad al jugador) tras una escena de mucha tensión para relajarlo. Contra uno de los enemigos finales llamado Psycho Mantis, cuya habilidad es leer la mente del jugador, el jugador debe adivinar que tiene que cambiar de ranura el controlador del juego (de jugador 1 a jugador 2) para evitar la “habilidad” de Psycho Mantis de leer sus pensamientos y por lo tanto de anticiparse a los mismos, siendo esta la única forma de derrotarlo. Este es sólo un sencillo ejemplo de los muchos que podemos encontrar donde las referencias entre el juego y la realidad se intercalan estimulando la sensación de inmersión del jugador.

\section{Realidad y emergencia de juego}

Los videojuegos utilizan sistemas dinámicos que hacen emerger situaciones diferentes en cada momento. Dichas situaciones están basadas en representaciones de la vida real, adaptándola a los requerimientos del juego. En Grand Theft Auto: San Andreas podemos circular por una ciudad llena de gente en la que continuamente están ocurriendo cosas a nuestro alrededor. La gente pasea por la ciudad, los policías hacen sus rondas, los coches circulan... y de repente un coche se sale de la calzada, intentando adelantar a una moto y atropella a un peatón. La gente grita y se acerca al herido, parece que alguien llama a una ambulancia y al momento podemos escuchar el sonido de la sirena de una ambulancia que se acerca; al llegar el conductor frena con un derrape y de la ambulancia bajan dos camilleros que se hacen cargo del 
herido, llevándoselo de nuevo entre sirenas al hospital. Todo esto ocurre en el juego sin que estuviera previsto en un guión y sin que el jugador haya intervenido en ningún momento.

Juuls (2004, pp. 80-81) habla de cuatro tipos de emergencia que afectan a las reglas de juego:

1. Emergencia como una variación: Son todos los estados posibles que permiten las reglas de un juego

2. Emergencia como una pauta: Las pautas de juego no se pueden deducir inmediatamente de las reglas de juego, ya que son demasiadas y pueden combinarse entre si de múltiples maneras.

3. Emergencia como irreductibilidad: Las reglas son muy definidas y no pueden combinarse entre si, por lo tanto sólo suele existir una manera de hacer las cosas en el juego.

4. Emergencia como novedad: varias reglas del juego se combinan de manera novedosa y surge un comportamiento nuevo.

En el juego emergente "el devenir de los acontecimientos secundarios puede adaptarse al jugador, aunque la historia principal siga necesitando del autor humano y no pueda ser alterada en sus acontecimientos principales” (Peinado y Santorum, 2004 p.1). Pueden utilizarse las ventajas del juego emergente en una narración basada en autor usando puntos de control para hacer avanzar el juego, "pero la manera de alcanzarlos dependerá de cada jugador, el sistema sólo se ocupa de verificar que se ha obtenido el resultado deseado, pero da libertad para llegar hasta él” (Peinado y Santorum, 2004 p.6).

Ahora bien, el juego emergente no es una nueva forma de narrar, es una nueva forma de crear juego combinando las reglas existentes: crea un entorno en el cual poder jugar, pero necesita la existencia de una historia y de un jugador para crear narración.

La posibilidad de combinar reglas en un universo diegético es la que llevará la noción de “emergencia” a un videojuego y creará una narración a la que se denomina “emergente”, esto es, dependiente de la combinación de reglas que genere el juego y las acciones del jugador. La ausencia de dicha posibilidad obligará a crear un juego que progresa convencionalmente y en el cual "avanzar” significará “aprender reglas nuevas” continuamente que, una vez utilizadas, no volverán a usarse pero que proporcionarán al autor un camino sólido y único para hacer avanzar al jugador a través del entorno de inmersión.

La narrativa emergente no soluciona el dilema interactivo planteado por Berenguer, en el cual la necesidad de controlar la historia por parte del autor se enfrenta a la necesidad de libertad del jugador (Berenguer, 1998), pero acerca al jugador la posibilidad de crear su propia historia dentro de unos márgenes elegidos por el autor.

A grandes rasgos, en los juegos convencionales - "progresivos” — (Juuls 2004), la jugabilidad se crea mediante excepciones, cada situación se debe resolver de forma particular. Sin embargo, en un juego emergente la jugabilidad debe estar diseñada mediante reglas que pueden combinarse creando pautas de 
comportamiento. Dichas pautas pueden aprenderse y facilitan el flujo del juego y de la historia, involucrando al jugador en el juego y en la historia al mismo tiempo, creando multitud de situaciones diferentes gracias a su arquitectura compartida, en la que muchos eventos pueden interactuar con otros, posibilitando la creación no de historias más complejas, sino de entornos más complejos y por lo tanto más reales.

En esta clase de juegos emergentes los diseñadores tienen unos rangos en los que pueden influir, el sistema se comporta de manera que es capaz de generar nuevas situaciones a partir de la combinación de eventos sencillos. Esto hace la experiencia del jugador más variada, pero ¿qué ocurre si esas situaciones se obtienen directamente del mundo real? ¿Cómo se crean mundos virtuales basados en elementos tomados de la realidad? ¿Qué implicaciones tiene esta práctica en el diseño de un videojuego emergente?

La emergencia sigue estando asegurada, pero la variedad de elementos a combinar se ve multiplicada gracias a la enorme cantidad de información que puede extraerse de la vida real, y por tanto la jugabilidad es mayor. Flight Simulator, por ejemplo, ofrece las dos alternativas: o bien seleccionar manualmente las condiciones meteorológicas que se desean experimentar o tomar directamente las condiciones meteorológicas reales de la región de vuelo.

La jugabilidad emergente en tiempo real usando elementos tomados de la realidad garantiza que ni el jugador ni los creadores del juego conozcan los datos que se está introduciendo en el juego, además de que en principio se puede asegurar una coherencia entre los elementos que se introduzcan (todo lo coherente que consideremos el mundo real). Toda emergencia, de por sí, alarga la vida del juego, y si además se toma la realidad como parámetro de entrada para el sistema de generación de contenidos (situaciones, personajes, información de todo tipo...), la fuente de jugabilidad se convierte en un recurso potencialmente inagotable.

\section{Internet y las bases de datos}

Fastr es un ejemplo de un juego sencillo que explota las ventajas de las grandes bases de datos, en este caso de imágenes. La aplicación web muestra una a una varias imágenes por pantalla, siendo todas ellas el resultado de hacer una búsqueda en Flickr con una sola palabra y el jugador tiene que adivinar cual es esa palabra en el menor tiempo posible o antes de que lo consigan otros jugadores. Es particularmente interesante cómo se utiliza un buscador para la función inversa de la que está pensado.

Minijuegos digitales como este son sólo la punta del iceberg del potencial que tiene Internet y otras enormes bases de datos disponibles en muchos casos para el público en general. Toda esa información bien aprovechada garantizará numerosos contenidos útiles (y directamente tomados de la realidad) para usar en videojuegos. Por ejemplo, la evolución de los juegos emergentes puede permitir crear réplicas de ciudades enteras con datos en tiempo real, tomados de las más diversas fuentes de información. 


\section{Conclusiones}

La rigidez que conlleva crear un videojuego con un principio, un final y una única manera de jugarlo contrasta con la variedad que puede alcanzarse utilizando un sistema emergente. Utilizar información real en un videojuego implica adaptar dinámicamente las reglas del mismo a las posibilidades que ofrezca esa información para prolongar la vida del juego de manera extraordinaria; pero como contrapartida podemos obtener en algunos casos un generador aleatorio e independiente muy difícil de controlar y tecnológicamente inmaduro (en el caso de los sistemas de Computación Afectiva y similar).

Existen multitud de páginas que proporcionan información en tiempo real sobre casi todos los campos que pueden ser utilizadas para crear juegos siempre cambiantes, que siempre representan un reto para el jugador (y desgraciadamente para el diseñador también) porque son diferentes en cada ejecución.

Nos gustaría compartir algunas reflexiones con el lector: ¿Cuánto falta para que se empiece a utilizar información pública (si el usuario da su permiso) como la que ya controla Google cuando le confiamos nuestro correo electrónico, nuestros calendarios, recopilaciones de enlaces favoritos, etc. Los personajes de ficción podrán dentro de nada llevar los nombres de nuestros amigos, los escenarios serán como nuestras ciudades, nuestros centros de ocio o de trabajo, se crearán perversiones de la realidad desconocidas hasta ahora, por estar más allá de la capacidad de creación de contenidos que posee un videojuego "per se”, por pertenecer todavía al terreno de la ciencia-ficción. Con respecto a la implicación de este fenómeno en los nuevos interfaces, los personajes de los juegos podrán adaptarse a las emociones, los sentimientos e incluso el temperamento y la personalidad de cada uno de los jugadores. Y por último, con respecto a la implicación funcional que más atención recibe en este trabajo (la realidad en el juego emergente) podemos asegurar que el introducir la realidad dentro de un juego hace que las posibilidades que se generan al combinar las reglas para formar una narración dependiente de las acciones del jugador mejoren la sensación de amplitud del mismo, porque el juego ofrece material distinto en cada ejecución.

Si aceptamos que la realidad puede tomar parte en juegos emergentes, y que esto va a ocurrir en un periodo de tiempo corto, más vale empezar a pensar en cómo utilizarla para ampliar a nuevos horizontes los contenidos de los juegos existentes, ampliando el flujo de información de la realidad hacia los videojuegos y viceversa, dando la posibilidad de crear sistemas con una alta rejugabilidad que además de entretener pueden realimentarse mutuamente con la vida real y nutrirse de su inmensa riqueza y diversidad. 


\section{Referencias}

\section{Bibliografía}

Annaud, Jean-Jacques (2001) Enemigo a las puertas / Enemy at the gates.

Aylett, Ruth (1999). Narrative in Virtual Environments - Towards Emergent Narrative. AAAI Symposium on Narrative Intelligence. pp 83-86

Berenguer, Xavier (1998). Historias por ordenador. Serra d'Or http://www.iua.upf.es/\%7Eberenguer/textos/histor/narrc.htm

Crawford, Chris (1982). The Art of Computer Game Design.

http://www.vancouver.wsu.edu/fac/peabody/game-book/Coverpage.html

Gilleade, Kiel Mark; Dix, Alan; Allanson, Jen (2005). Affective Videogames and Modes of Affective Gaming: Assist Me, Challenge Me, Emote Me. Proceeding of 2nd International Conference of the Digital Games Research Association (DIGRA). Vancouver, Canada.

http://hdl.handle.net/1892/1603

Juul, Jesper (2004). Half Real, Cambridge, MIT Press.

Ryan, Marie-Laure (1992). Possible Worlds, Artificial Intelligence, and Narrative Theory.

\section{Ludografía}

Animal Crossing (2004) Nintendo.

http://www.animal-crossing.com

Battlefield 1942, Desert Combat (2004) Trauma Studios.

http://www.desertcombat.com/

Call of Duty 2 (2005) Infinity Ward. Activision.

http://www.callofduty.com/

Canyouseemenow (2005) Blast Theory, Mixed Reality Lab and Equator.

http://www.canyouseemenow.co.uk

Catechumen (2001) Centurion Soft / N'Lightning Software.

http://www.catechumen.co.uk/

Crysis (previsto para 2007) Crytek Studios. Electronic Arts.

http://www.es.ea.com/games/8524/

EA Sports. Electronic Arts.

http://es.easports.com/ 
Everquest (1999) Sony Online Entertainment.

http://everquest.station.sony.com/

Fastr (2006) RadomChaos.

http://www.randomchaos.com/games/fastr/es/

Flight Simulator 2004. A Century of Flight (1981-2004) Microsoft.

http://www.microsoft.com/games/flightsimulator/

Food Force (2005) United Nations.

http://www.food-force.com/

Grand Theft Auto: San Andreas (2005) Rock Star.

http://www.gta-sanandreas.com/

Half-Life 2 (2004) Valve Software.

http://half-life2.com/

Jungle Warrior (1990) Zigurat.

Kuma\War and Kuma\Reality Games (2005) Kuma.

http://www.kumawar.com/

McDonalds Videogame (2006)

http://www.mcvideogame.com

Metal Gear Solid (1998) Konami.

http://www.konami.jp/gs/game/metalgear/

Myst, (1993) Cyan

http://www.cyan.com/

oGame (2004) Gameforge GmbH

http://ogame.com.es/

Paris Riots (2006)

http://parisriots.free.fr/

September 12th (2003) Newsgaming.

http://www.newsgaming.com/games/index12.htm

Shadow of Memories (2001) Konami.

http://uk.konami-europe.com/game.do?idGame=44\&idGamePlatformInfo=63

Tetris (1985) Alexey Pajitnov.

http://www.tetris-today.com/story/original-tetris0.shtml

Ultima Online (1997) Origin Systems. Electronic Arts.

http://www.uo.com/ 
V-Girl, (2004) Artificial Life

http://www.v-girl.com/

World of Warcraft (2005) Blizzard Entertainment

http://www.worldofwarcraft.com/ 